\section{Presentation and incidence of Hirschsprung's disease}

Hirschsprung's disease is a well recognised cause of obstruction in neonates, but considerable delay may still occur in diagnosing it in older children. In south east Scotland (Lothians, Borders, and Fife) all children noted to have constipation from the first year of life who fail to respond to conventional treatment are now investigated by anorectal manometry and rectal mucosal biopsies. Thus $17 \%$ of these patients have been found to have Hirschsprung's disease, which is a dramatic increase ir the incidence of diagnosis, particularly in older children and those with ultrashort segment disease. ${ }^{1}$ As the exact incidence and pattern of presentation of Hirschsprung's disease in the United Kingdom are unknown ${ }^{2}$ these findings prompted a study of the local population to establish the incidence of the disease, its familial occurrence, and its pattern of presentation within the community.

\section{Patients, methods, and results}

We reviewed the case records of 103 patients with Hirschsprung's disease born in south east Scotland during the 30 years 1953-82. We obtained additional information from the patients' families and their general practitioners. The length of aganglionic segment was described as ultrashort (distal third of rectum or less) in 15 patients; short (rectum or rectum and sigmoid colon) in 69; and long (proximal colonic disease) in 19. There were 82 male and 21 female patients, a ratio of $4: 1$. Apart from five patients with total colonic aganglionosis, in whom the sex ratio was $1 \cdot 5: 1$, there was no variation in the sex incidence with varying lengths of aganglionic bowel. The table shows the age at diagnosis.

Age at diagnosis of Hirschsprung's disease

\begin{tabular}{lccc}
\hline & \multicolumn{3}{c}{ Year of birth } \\
\cline { 2 - 3 } Age at diagnosis & $1953-67$ & $1967-82$ & Total \\
\hline Neonate & 27 & 28 & 55 \\
< years & 7 & 17 & 24 \\
5-14 years & 6 & 18 & 24 \\
\hline Total & 40 & 63 & 103 \\
\hline
\end{tabular}

The 103 patients came from 98 families, giving a family incidence of $6 \%$ Of 238 siblings traced, five had the disease, giving an incidence of $2 \%$. From the reports of the Registrar General in Scotland for the first 25 years of the study we established the incidence of the disease in this geographical area as being one in 4500 live births (99/445 000).

\section{Comment}

This is the first regional study of Hirschsprung's disease in the United Kingdom. Previous genetic studies have recorded information on patients drawn from a wide area with poorly defined populations. ${ }^{3}$ We had an advantage in that patients were referred to a single surgeon (WGS) from a well defined geographical area; this offered the ideal basis from which to study the pattern and incidence of a disease. With an established incidence of one in $\mathbf{4 5 0 0}$ live births, 140 new cases of Hirschsprung's disease may be expected each year in the United Kingdom. With an established family incidence of $6 \%$ it is essential that at the time of diagnosis an accurate family history is obtained so that potential patients may be traced.

In 1964 Bentley first described ultrashort segment Hirschsprung's disease, ${ }^{4}$ which is often diagnosed late in older children who have previously been described as encopretic or suffering from psychological problems. ${ }^{1}$ Indeed, almost half of the patients in this series in whom the disease was diagnosed late had ultrashort segment disease. Awareness that some of these patients may have a surgically correctible lesion permits referral for initial investigation by anorectal manometry and if indicated rectal mucosal punch biopsy. Histochemical analysis of the biopsy sample for acetyl cholinesterase yields rapid confirmation of the diagnosis. ${ }^{5}$ After the introduction of these measures the disease will be diagnosed in an increased number of patients, but the table shows that this increase will occur mainly in older children. Most patients should still be diagnosed as neonates, but an awareness of the problem in older children allows earlier referral for diagnosis and treatment.

${ }^{1}$ Scobie WG, MacKinlay GA. Ano-rectal myectomy in treatment of ultrashort segment Hirschsprung's disease. Arch Dis Child 1977;52:713-5. ${ }^{2}$ Lister J, Rickham PP. Hirschsprung's disease. In: Rickham PP, Lister J, Irving IM, eds. Neonatal surgery. 2nd ed. London: Butterworth, 1978: $441-5$

${ }^{3}$ Bodian M, Carter CO. A family study of Hirschsprung's disease. Ann Hum Genet 1963;26:261-77.

4 Bentley JFR. Some new observations on megacolon in infancy and childhood with special reference to the management of megasigmoid and megarectum. Dis Colon Rectum 1964;7:462-70.

${ }^{5}$ Hamdi MH, Scobie WG, Smith I, Patricks A. The changing position of punch biopsy of the rectum combined with acetyl cholinesterase study in the management of chronic constipation in childhood. $Z$ Kinderchir $1979 ; 26: 124-32$.

(Accepted 31 August 1983)

Surgical Paediatric Department, Western General Hospital, Edinburgh EH4 2XU

J D ORR, MB, FRCs, senior surgical registrar

W G SCOBIE, MB, FRCS, consultant paediatric surgeon

Correspondence to: $\mathrm{Mr}$ J D Orr.

\section{Intestinal perforation associated with osmotic slow release indomethacin capsules}

Patients presenting with bleeding or perforation due to a peptic ulcer have often recently taken non-steroidal anti-inflammatory drugs. The introduction of a new formulation of indomethacin (Osmosin) designed to release the drug in the small intestine should decrease the risk of these complications, but it may result in complications affecting the small bowel and colon. We describe two patients who suffered such complications.

\section{Case reports}

Case 1-A 70 year old man had been taking ordinary indomethacin tablets $25 \mathrm{mg}$ three times daily for 10 years. Two weeks before admission with severe diarrhoea he had been changed to slow release indomethacin trihydrate 105 mg twice daily. He had had a gastrectomy for duodenal ulcer in 1957. On admission he was found to be mildly dehydrated, and was initially diagnosed as having infective diarrhoea. Faecal cultures, however, were negative for pathogens, sigmoidoscopic appearances were normal, and his condition appeared to resolve with rehydration. Three days after admission he became confused. He did not complain of abdominal pain but his abdomen was found to be rigid. He was apyrexial, not shocked, and maintained a constant pulse rate of 110 beats/minute. $X$ ray examinations of the abdomen showed copious free gas. At operation he was found to have multiple haemorrhagic perforating ulcers of the terminal ileum. Adjacent to the perforation were two capsules of slow release indomethacin. A further capsule in the ascending colon was adjacent to a small area of ulceration. Cultures of faeces and mesenteric lymph nodes were negative for Salmonella spp. After operation he developed further ulceration and perforation of the ileum and colon, from which he died two days later. At necropsy a further capsule of slow release indomethacin was recovered from the abdominal cavity.

Case 2-A 79 year old woman with a longstanding history of diverticular disease was admitted with an exacerbation of abdominal pain. She had been taking benorylate $4 \mathrm{~g}$ twice daily for osteoarthritis, and two weeks before admission this had been supplemented by slow release indomethacin trihydrate $105 \mathrm{mg}$ twice daily. On examination she was apyrexial with a pulse rate of 90 beats/minute and a blood pressure of $140 / 90 \mathrm{~mm} \mathrm{Hg}$. Her abdomen was diffusely tender and almost rigid. Bowel sounds were absent. Laboratory findings were: haemoglobin $12 \cdot 1 \mathrm{~g} / \mathrm{dl}$, while blood cell count $6.7 \times 10^{\circ} / 1$, and serum amylase $105 \mathrm{IU} / 1$ (normal $70-300 \mathrm{IU} / 1) . X$ ray examination of the chest and abdomen showed nothing abnormal. Abdominal paracentesis showed a purulent peritonitis. Metronidazole $500 \mathrm{mg}$ and cephazolin sodium $1 \mathrm{~g}$ were instilled via the paracentesis catheter, and she was rehydrated with intravenous fluids. At operation she had faecal peritonitis. There was a $4 \mathrm{~mm}$ 